\title{
Dénombrements directs des bactéries des milieux aquatiques par microscopie en épifluorescence : comparaison entre un système d'analyse d'images automatisé (Mudicam $\left.{ }^{\circledR}\right)$ et l'observation visuelle \\ Direct count of aquatic bacteria by epifluorescence microscopy : comparison of visual and image analyser (Mudicam ${ }^{\circledR}$ ) techniques
}

\author{
P. Got, B. Baleux et M. Troussellier
}

Volume 6, numéro 3, 1993

URI : https://id.erudit.org/iderudit/705176ar

DOI : https://doi.org/10.7202/705176ar

Aller au sommaire du numéro

Éditeur(s)

Université du Québec - INRS-Eau, Terre et Environnement (INRS-ETE)

ISSN

0992-7158 (imprimé)

1718-8598 (numérique)

Découvrir la revue

Citer cet article

Got, P., Baleux, B. \& Troussellier, M. (1993). Dénombrements directs des bactéries des milieux aquatiques par microscopie en épifluorescence : comparaison entre un système d'analyse d'images automatisé (Mudicam®) et l'observation visuelle. Revue des sciences de l'eau / Journal of Water Science, 6(3), 269-284. https://doi.org/10.7202/705176ar

\section{Résumé de l'article}

La technique de comptage par microscopie en épitluorescence est la méthode la plus performante pour dénombrer la totalité des bactéries présentes dans les milieux aquatiques. Cependant cette technique est longue, fastidieuse et subjective. Afin d'automatiser et de rendre objectif le dénombrement, le microscope à épifluorescence est couplé à un analyseur d'images. Si les systèmes d'analyse d'images sont utilisés pour les mesures de taille des bactéries aquatiques, très peu d'études font état de comparaison entre les dénombrements par analyse d'image et ceux réalisés de façon traditionnelle. Cet article présente les résultats des dénombrements de souches bactériennes de référence et de bactéries des milieux aquatiques, par la technique de microscopie en épifluorescence des cellules bactériennes marquées au DAPI, réalisés simultanément par observation microscopique visuelle (visuel) et par analyse d'images automatisée (automatique).

Le système d'analyse d'images est composé d'une caméra vidéo (Lhesa LH40036) de sensibilité de $510^{-4}$ lux, d'une carte de numérisation ( 512 x 512 pixels, 8 bits, cyclope v 2.32, Digital vision) d'un micro-ordinateur 80-386 et d'un logiciel de dénombrement (Mudicam ${ }^{\circledR}$. EAU). Le système est couplé à un microscope en épilluorescence Olympus BH2.Les dénombrements ont été réalisés d'une part sur des suspensions de souches bactériennes de référence $(n=30)$ à différents états physiologiques et sur des échantillons d'eaux $(n=50)$ d'origines diverses (fleuve, eaux saumâtre, marine et résiduaire). La comparaison des deux méthodes est réalisée par un modèle de régression linéaire et une analyse de variance. Les tests statistiques associés permettent de conclure à une bonne concordance entre les deux méthodes. A partir de l'ensemble des dénombrements réalisés, 18 d'entre eux pris au hasard ont été dénombrés de façon manuelle par deux opérateurs et par le système d'analyse d'image. Il apparaît que les différences de comptage les plus élevées correspondent aux dénombrements effectués par chacun des deux opérateurs Ceci met en évidence que non seulement le système d'analyse d'image permet une quantification rapide des abondances bactériennes, mais en outre il supprime la subjectivité de l'opérateur tout en réalisant des dénombrements aussi précis.
Ce document est protégé par la loi sur le droit d'auteur. L’utilisation des services d’Érudit (y compris la reproduction) est assujettie à sa politique d'utilisation que vous pouvez consulter en ligne.

https://apropos.erudit.org/fr/usagers/politique-dutilisation/ 


\title{
Dénombrements directs des bactéries des milieux aquatiques par microscopie en épifluo- rescence : comparaison entre un système d'analyse d'images automatisé (Mudicam ${ }^{\circledR}$ ) et l'observation visuelle
}

\author{
Direct count of aquatic bacteria by epifluorescence \\ microscopy : comparison of visual and image analyser \\ (Mudicam®) techniques
}

\section{P. GOT1, B. BALEUX1, M. TROUSSELLIER ${ }^{1}$}

Reçu le 3 février 1993, accepté pour publication le $1^{\text {er juin } 1993 . *}$

SUMMARY

Direct counting by epifluorescence microscopy is the best method available to determine total counts of aquatic bacteria. However, microscopic observation is tedious and time-consuming. A more rapid and certainly less subjective way of counting bacteria is to combine epifluorescence microscopy with an image analysis system. Surprisingly, although image analysis is now a relatively common method to measure the size of aquatic bacteria, very few studies have been devoted to the validation of total counts by image-analysis systems. In this paper, we present data on simultaneous determination of total counts of 4'6-diamidino-2-phenylindole (DAPI) stained bacteria by visual means and by image-analysed (Mudicam ${ }^{\circledR}$ system) epifluorescence microscopy methods.

The Olympus microscope BHZ is equipped for epifluorescence with a $100 \mathrm{~W}$ $\mathrm{Hg}$ lamp and a 100x oil immersion objective (Apo UVFL 160/1.3). The image analysis system consists of a high performance $\left(5 \times 10^{-6}\right.$ lux $)$ video camera (Lhesa LH40036) and an Image processor which digitalizes the video image in a grey scale extending from 0 (black) to 255 (white) into a binary image with $512 \times 512$ pixels (8 bit, cyclope v 2.32, Digital Vision), and image analysis software (MUDICAM\&. EAU). The samples were stained with DAPI (final concentration $2.5 \mu \mathrm{g} / \mathrm{ml})$ and filtered through polycarbonate filters $(0.22 \mu \mathrm{m}$, Nuclepore Corporation). The surface area of the video image is $76 \times 111 \mu \mathrm{m}^{2}$.

The analysed samples come from culture collections of different bacterial strains $(n=30)$ submitted to different conditions and incubation times to obtain various physiological states (Table 1). The natural water samples were collected from several aquatic ecosystems: Rhóne river, Mediterranean sea, Thau lagoon and Montpellier sewage waters $(n=50)$. The bacterial abundances ranged from $10^{5}$ to $10^{8} \mathrm{cellis} / \mathrm{ml}$ and the size range of the cells varied from 0.63 to $17 \mu \mathrm{m}^{2}$.

1. Laboratoire d'Hydrobiologie Marine, U.R.A. CNRS 1355, Université Montpellier II 34095 Montpellier cedex 5.

* Les commentaires seront reçus jusqu'au 31 mars 1994. 
Comparisons between the image analysis and visual counts were made on the basis of thirty fields per filter. The image analysis counts are based on a two step procedure. The video image of each microscopic field is first numerised and stored on a hard disk (153 Mo). When all the fields have been stored, the digitized images are submitted to an automatic thresholding which allows background substraction. Automatic counting of bacterial cells is then performed on the basis of object specifications defined by the operator. These specifications concern the minima and maxima values of the area (expressed in pixel numbers) and the fluorescence (expressed in grey levels) of the objects. The MUDICAM@EAU software also provides the mean number of cells per millilitre and the associated variance.

Average concêntrations and confidence limits are shown in Table 2 for bacterial collection strain cultures and in Table 3 for water samples. When we compared visual and image analysis counts by linear regression, the ability of the image analysis system to enumerate bacterial cells was clearly demonstrated. With bacterial culture (Fig. 2) and with water samples (Fig. 3), the coefficients of correlation were respectively $r=0.997$ and $r=0.996$ $(p=0.0001)$. The slopes of the models are not significantly different from unity and the $Y$-intercepts are not different from zero. Moreover we have compared the total visual counts of two experimenters and the image-analysed counts on eighteen random samples (Table 4). The variance analysis shows that there is no difference between the three methods, with mean value of $6.09,6.08$ and 6.11 for the image-analysed method, experimenter $n^{\circ} 1$ and experimenter $n^{\circ} 2$, respectively. While non significant, the greatest difference in counts was obtained between the two experimenters.

It may be concluded that the image analyser tested for total counts by epifluorescence microscopy is a precise and rapid procedure for the determination of total bacterial counts. This method may be standardized and its automation allows the analysis of many samples, an important advantage in ecological studies. Storage of the samples also allows one to treat a posteriori some complementary aspects of the total count, such as the double staining of bacteria. The image analyser tested is appropriate for bacterial ecology studies which require epifluorescence microscopy.

Key-words : bacterial count, epifluorescence microscopy, image analysis, bacterial collection strains, aquatic environmental bacteria, DAPI.

RÉSUMÉ

La technique de comptage par microscopie en épifiluorescence est la méthode la plus performante pour dénombrer la totalité des bactéries présentes dans les milieux aquatiques. Cependant cette technique est longue, fastidieuse et subjective. Afin d'automatiser et de rendre objectif le dénombrement, le microscope à épifluorescence est couplé à un analyseur d'images. Si les systèmes d'analyse d'images sont utilisés pour les mesures de taille des bactéries aquatiques, très peu d'études font état de comparaison entre les dénombrements par analyse d'image et ceux réalisés de façon traditionnelle. Cet article présente les résultats des dénombrements de souches bactériennes de référence et de bactéries des milieux aquatiques, par la technique de microscopie en épifluorescence des cellules bactériennes marquées au DAPI, réalisés simultanément par observation microscopique visuelle (visuel) et par analyse d'images automatisée (automatique).

Le système d'analyse d'images est composé d'une caméra vidéo (Lhesa

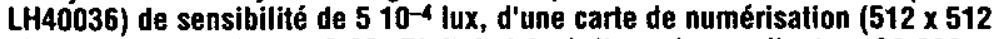
pixels, 8 bits, cyclope v 2.32, Digital vision) d'un micro-ordinateur $80-386$ et d'un logiciel de dénombrement (Mudicam® $\otimes$. EAU). Le système est couplé à un microscope en épifluorescence Olympus BH2. 
Les dénombrements ont été réalisés d'une part sur des suspensions de souches bactériennes de référence $(n=30)$ à différents états physiologiques et sur des échantillons d'eaux ( $n=50)$ d'origines diverses (fleuve, eaux saumâtre, marine et résiduaire). La comparaison des deux méthodes est réalisée par un modèle de régression linéaire et une analyse de variance. Les tests statistiques associés permettent de conclure à une bonne concordance entre les deux méthodes. A partir de l'ensemble des dénombrements réalisés, 18 d'entre eux pris au hasard ont été dénombrés de façon manuelle par deux opérateurs et par le système d'analyse d'image. II apparaît que les différences de comptage les plus élevées correspondent aux dénombrements effectués par chacun des deux opérateurs. Ceci met en évidence que non seulement le système d'analyse dimage permet une quantification rapide des abondances bactériennes, mais en outre il supprime la subjectivité de l'opérateur tout en réalisant des dénombrements aussi précis.

Mots clés : Dénombrements bactériens, microscopie en épifluorescence, analyse d'images, souches bactériennes de référence, bactéries des milieux aquatiques, DAPI.

\section{1 - INTRODUCTION}

La technique du comptage par microscopie en épifluorescence est très largement utilisée et reconnue comme étant la plus performante pour dénombrer la totalité des cellules bactériennes présentes dans les milieux aquatiques (ROBARTS and SEPHTON, 1981 ; FRY, 1988 ; SIERACKI et al., 1989 ; HARA et al.,1991). La précision de ces dénombrements est fonction du nombre de champs microscopiques comptés et du nombre moyen de bactéries par champs (TROUSSELLIER et al., 1985). L'automatisation au moyen d'un système d'analyse d'images du comptage par microscopie en épifluorescence a pour but d'affranchir l'opérateur de la fatigue visuelle qui est le principal facteur limitant du nombre d'échantillon analysé. Cette automatisation doit offrir également un gain de temps non négligeable dans le dénombrement des cellules bactériennes de l'échantillon permettant ainsi d'augmenter le nombre d'analyses (VECHT-LIFSHITZ and ISON, 1992). Elle doit permettre enfin d'évaluer de façon reproductible les abondances bactériennes contenues dans les échantillons sans prendre en compte la subjectivité et la variabilité inhérente à l'opérateur.

Le premier procédé de comptage de bactéries par microscopie en épifluorescence utilisant un analyseur d'images a été mis au point pour le dénombrement des bactéries du lait (GRAPPIN et JEUNET, 1974 ; PETTIPHER and RODRIGUES, 1982) puis étendu à d'autres produits alimentaires (ABGRALL et BOURGEOIS, 1989 ; DASEN et al., 1989 ; JAEGGI et al., 1989) mais tous concernent le comptage de cellules bactériennes présentant une taille et une intensité de fluorescence très supérieures à celles rencontrées pour les cellules bactériennes de l'environnement aquatique.

Si des appareillages de dénombrements automatiques tels que ceux faisant appel à l'analyse d'images ont été développés dans le domaine ali- 
mentaire c'est par suite des enjeux importants que présente le contrôle de qualité bactériologique dans l'industrie agro-alimentaire. Les appareils adaptés au dénombrement total des bactéries dans les eaux permettent soit la numération " des particules les plus fluorescentes " dans des eaux embouteillées et ces particules sont comptabilisées comme étant des bactéries (OGER et al., 1987), soit le comptage des cellules bactériennes de référence ou dont les dimensions ont été augmentées artificiellement par l'action de substances antibiotiques (acide nalidixique) bloquant la division cellulaire (SINGH et al., 1990 ; CHERYL and DAVIES, 1991). La petite taille et le faible niveau de fluorescence des cellules bacjériennes présentes dans la plupart des milieux aquatiques naturels nécessitent l'utilisation d'un matériel suffisamment sensible et adapté à leurs dénombrements et dont peu de descriptions existent. Les quelques études réalisées font référence à des appareils dont la conception fait appel à un assemblage artisanal des composants du système (BJORNSEN, 1986 ; VAN WAMBEKE, 1988) avec des logiciels informatiques non commercialisés. De plus elles ne présentent des validations des dénombrements automatiques que sur quelques champs microscopiques voire au mieux sur une dizaine d'échantillons (SIERACKI et al., 1985).

Le but de cet article est de présenter les résultats des dénombrements des cellules bactériennes par microscopie en épifluorescence au moyen d'un système d'analyse d'image nouvellement commercialisé, en comparaison au comptage par observation microscopique visuelle. Les essais ont été réalisés sur différentes souches bactériennes de référence et sur divers échantillons d'eaux de milieux aquatiques naturels continentaux ou marins et d'eaux résiduaires épurées ou non.

\section{2 - MATÉRIEL ET MÉTHODES}

\subsection{Origine des échantillons}

Les 11 souches bactériennes de référence utilisées dans cette étude sont présentées dans le Tableau 1. Elles regroupent des cellules présentant des états physiologiques (24 h, $48 \mathrm{~h}, 7$ j, 10 j, 5 mois), des tailles $\left(0,63\right.$ à $\left.17 \mu \mathrm{m}^{2}\right)$, des morphologies (cocci, bacilles) différents et des conditions d'incubations (gélose et bouillon nutritif bioMérieux, eau physiologique à $9 \mathrm{gl}^{-1} \mathrm{Cl} \mathrm{Na}$ ) variées, soit 30 essais.

Les échantillons d'eaux issus des milieux aquatiques, proviennent de diverses origines: (Tableau 3) eaux saumâtres ( $n^{\circ} 101$ à 115 : étang de Thau), marines ( $n^{\circ} 116$ à 126: Méditerranée), continentales ( $n^{\circ} 127$ à 130 : fleuve Rhône, $n^{\circ}$ 131-132: fleuve Hérault), résiduaires ( $n^{\circ} 133$ à 135 : eau d'égout, $n^{\circ} 136$ à 149 : rejet de station d'épuration biologique, $n^{\circ} 150$ : rejet de station de lagunage). 
Tableau 1 Numérotation, nom, origine, (ATCC: American Type Culture Collection, IUT : Institut Universitaire de Technologie Montpellier II Département de biologie appliquée), milieu (GN : gélose nutritive, BN : bouillon nutritif, EPh : eau physiologique) et temps d'incubation des souches bactériennes de référence. $\$$ : échantillon traité aux ultra-sons.

Table 1 Number designation, name, origin (ATCC: American Type Culture Collection, IUT: Institut Universitaire de Technologie Montpellier II Département de biologie appliquée), isolation medium, (GN : nutrient agar; BN: nutrient broth; EPh : physiological water) and incubation time of bacterial culture collection strains. $\$$ : sonicated samples.

\begin{tabular}{|clccc|}
\hline \multicolumn{1}{|c}{ Souches } & & $\begin{array}{c}\text { Mlliou } \\
\text { d'incubation }\end{array}$ & $\begin{array}{c}\text { Temps } \\
\text { d'Incubation }\end{array}$ \\
\hline 1 & Citrobacter freundii & IUT & GN & $24 \mathrm{~h}$ \\
2 & Proteus vulgaris & ATCC13315 & GN & $24 \mathrm{~h}$ \\
3 & Salmonella arizonae & IUT & GN & $24 \mathrm{~h}$ \\
4 & Salmonella typhimurium & IUT & GN & $24 \mathrm{~h}$ \\
5 & Proteus vulgaris & ATCC13315 & GN & $48 \mathrm{~h}$ \\
6 & Escherichia coli 1832 & IUT & GN & $48 \mathrm{~h}$ \\
7 & Serratia marcescens & ATCC13880 & GN & $48 \mathrm{~h}$ \\
8 & Escherichia coli 0126B16 & ATCC14948 & EPh & $24 \mathrm{~h}$ \\
9 & Serratia marcescens & ATCC13880 & BN & $24 \mathrm{~h}$ \\
10 & Escherichia coli 0126B16 & ATCC14948 & BN & $24 \mathrm{~h}$ \\
11 & Serratia marcescens & ATCC13880 & EPh & $24 \mathrm{~h}$ \\
12 & Escherichia coli 0126816 & ATCC14948 & GN & $24 \mathrm{~h}$ \\
13 & Escherichia coli 0126B16 & ATCC14948 & GN & $24 \mathrm{~h}$ \\
14 & Escherichia coli 0126B16 & ATCC14948 & GN & $24 \mathrm{~h}$ \\
15 & Escherichia coli 0126B16 & ATCC14948 & GN & $24 \mathrm{~h}$ \\
16 & Escherichia coli 0126B16 & ATCC14948 & GN & $24 \mathrm{~h}$ \\
17 & Escherichia coli 0126B16 & ATCC14948 & EPh & 10 jours \\
18 & Bacillus megaterium & ATCC14581 & BN & 10 jours \\
19 & Serratia marcescens & ATCC13880 & BN & 7 jours \\
20 & Serratia mancescens & ATCC13880 & BN & 7 jours \\
$21 \$$ & Escherichia coli 0128B12 & IUT & GN & $24 \mathrm{~h}$ \\
22 & Escherichia coli 0128B12 & IUT & GN & $24 \mathrm{~h}$ \\
$23 \$$ & Staphylococcus aureus & ATCC12600 & GN & $24 \mathrm{~h}$ \\
24 & Escherichia coli 0126B16 & ATCC14948 & GN & $48 \mathrm{~h}$ \\
$25 \$$ & Streptococcus faecalis & IUT & GN & $24 \mathrm{~h}$ \\
$26 \$$ & Aeromonas hydrophila & ATCC15467 & GN & $24 \mathrm{~h}$ \\
$27 \$$ & Citrobacter freundii & IUT & BN & $24 \mathrm{~h}$ \\
28 & Salmonella typhimurium & IUT & GN & $24 \mathrm{~h}$ \\
29 & Escherichia coli 0126B16 & ATCC14948 & BN & $5 \mathrm{mois}$ \\
30 & Escherichia coli 0126B16 & ATCC14948 & EPh & $5 \mathrm{mois}$ \\
\hline & & & & \\
\hline
\end{tabular}




\subsection{Préparation des échantillons}

Les échantillons sont fixés par une solution de formaldéhyde tamponnée à la concentration finale de $4 \%$ (PORTER et FEIG, 1980) et conservés à l'obs curité à $4{ }^{\circ} \mathrm{C}$ pendant une durée ne dépassant pas quinze jours.

Compte tenu du caractère très agrégatif de certaines souches bactériennes de référence un traitement dans un bain à ultra-sons de $5 \mathrm{mn}$ à $75 \mathrm{~W}$ a été appliqué avant marquage sur leur suspension bactérienne. Les numéros de ces souches sont suivis du sigle: “\$”(Tableau 1).

La coloration des bactéries se fait par un fluorochrome, le 4',6-Diamidino-2-phényl-indole, (DAPI, Sigma). Ce dernier est plus performant que le premier fluorochrome utilisé en bactériologie à savoir l'orangé d'acridine (STRUGGER, 1948) pour dénombrer les bactéries (ROBARTS and SEPHTON, 1981) car il offre une plus grande stabilité (YANAGIDA et al., 1986) et une plus grande intensité de fluorescence tout en présentant une grande affinité pour les bases A-T de I'ADN (KING and PARKER, 1988). La concentration finale de DAPI dans l'échantillon ou sa dilution (tampon tris $\mathrm{pH}=7,1$ ) est de $2,5 \mu \mathrm{g} / \mathrm{ml}$ (ROBERTSON and BUTTON, 1989). Après un temps de contact de $15 \mathrm{mn}$ à la température ambiante et à l'obscurité, l'échantillon ainsi coloré est filtré sous vide $(-127 \mathrm{~mm} \mathrm{Hg})$ au travers d'une membrane en polycarbonate noire (Nuclépore, inc. corporation) de diamètre $25 \mathrm{~mm}$ et de porosité $0,2 \mu \mathrm{m}$. Cette membrane est montée entre lame et lamelle avec de l'huile à immersion non fluorescente (Olympus) et placée sous le microscope.

\subsection{Microscope et analyseur d'images}

Le microscope de type $\mathrm{BH} 2$ (Olympus) est équipé d'un système à épifluorescence avec lampe à vapeur de mercure HBO $100 \mathrm{~W}$ (Osram), d'un miroir dichroïque DMUB (BP340, LP420nm) et d'un objectif à immersion UVFL $x 100 / 1,30$ ( $i=1,404$ Olympus).

Le système d'analyse d'image Mudicam® (IRTN', Fig. 1) est composé d'une caméra de sensibilité de $51^{-4}$ lux (LHESA LH40036) qui transmet les images vidéo captées par le tube à une carte de numérisation (cyclope $\vee 2.32$, Digital vision). Les images numérisées sont visualisées sur un écran dont la définition est de $512 \times 512$ pixels et 256 niveaux de gris. Le micro-ordinateur gérant le système est composé d'un coprocesseur 80-386 compatible IBM PC A-T cadencé à 25 méga-hertz avec une mémoire vive de 4 mégaoctets et une mémoire de masse de 153 mégaoctets. La surface d'un point image obtenu ou pixel est de $0,2 \times 0,15 \mu \mathrm{m}$ avec l'objectif $\times 100$ et l'oculaire caméra 3,3 . La surface du champ microscopique analysé par la caméra est de $76 \times 111 \mu \mathrm{m}$.

Le protocole de numération consiste de façon générale, pour chaque échantillon, à dénombrer par observation visuelle et de façon automatique par l'analyseur 30 champs microscopiques choisis au hasard sur la préparation. Pour certains échantillons issus des milieux aquatiques (Tableau 3) seuls 10 champs ont été dénombrés par chacune des deux méthodes. Après stockage

1. IRTN S.A., 26 rue Théron de Montaugé, Z.I. de Gramont, 31200 Toulouse FRANCE. 


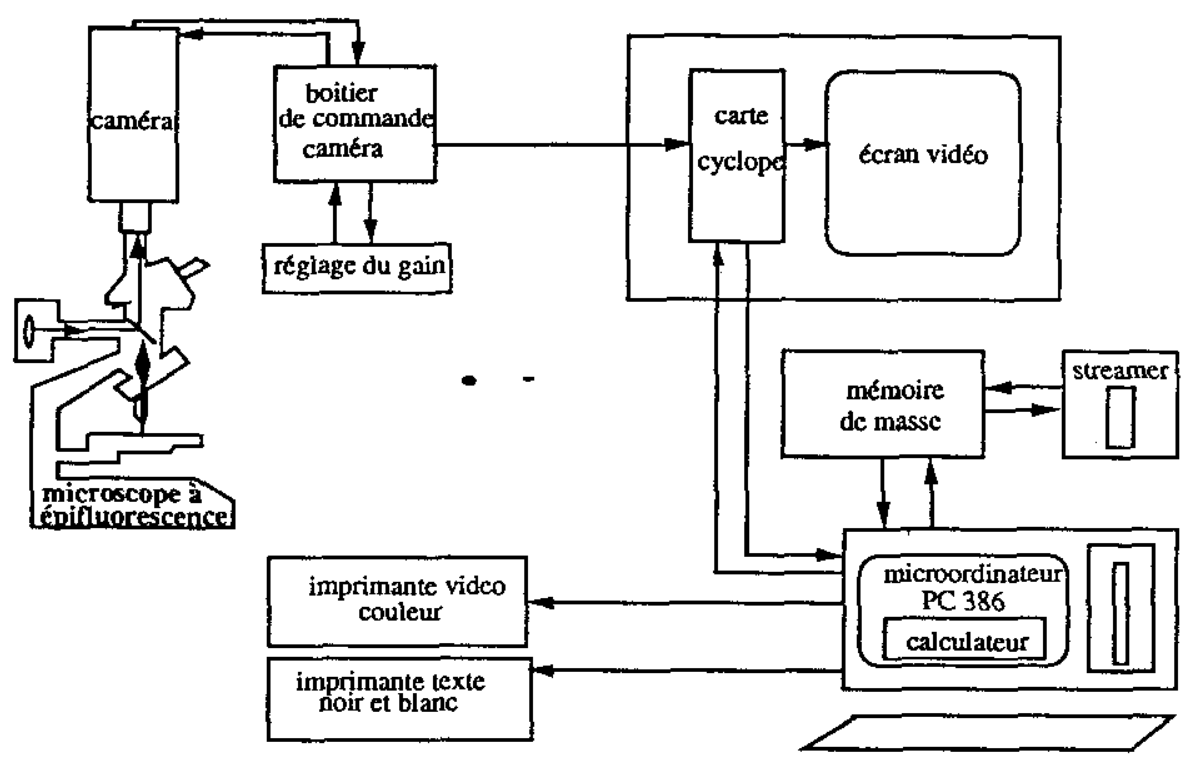

Figure 1 Schéma synoptique du système d'analyse d'images (Mudicam®). Diagram of the image analysis system (Mudicam®).

des images numérisées correspondant aux champs microscopiques les images sont traitées par le logicie! Mudicam®. EAU. Elles subissent dans un premier temps une mise à seuil automatique dont le but est d'éliminer par "filtrage " le bruit de fond de la préparation (autofluorescence résiduelle de la membrane par exemple) tout en conservant les petites cellules de faible fluorescence. Dans un second temps l'utilisation de commandes de programme (que l'opérateur a préalablement définies sur la base des valeurs des caractéristiques des cellules à dénombrer: surface, luminescence) permet d'aboutir aux estimations de la concentration moyenne des bactéries par unité de volume et de la variance associée.

\subsection{Méthodes statistiques}

La moyenne $(x)$ de chaque échantillon est calculèe à partir des n champs microscopiques dénombrés et la variance associée $\left(S^{2}\right)$ permet de définir l'intervalle de confiance au seuil de $95 \%$ autour de la moyenne.

Le mode de répartition des cellules sur le filtre est évalué par le calcul d'un indice de dispersion (l) qui correspond au rapport de la variance sur la moyenne de chaque échantillon $\left(I=S^{2} / x\right)$. Cet indice s'approche de 1 lorsque la distribution des cellules est aléatoire. Pour tester si les distributions observées pour chaque échantillon s'écarte ou non d'une distribution aléatoire, f'expression I ( $n-1)$, est calculée en partant du principe qu'elle correspond à une bonne approximation de $\mathrm{Khi}^{2}$ avec $(\mathrm{n}-1)$ degrés de liberté 
(ELLIOTT, 1977). Lorsque I ( $n$ - 1) est plus grand que la valeur attendue en référence à une table de $\mathrm{Khi}^{2}$ au seuil de $95 \%$ de signification, la distribution est considérée comme non aléatoire et contagieuse. En cas de distribution contagieuse, l'échantillon est soumis à un traitement par ultra-sons (voir plus haut).

Un modèle de régression linéaire simple est utilisé afin de mettre en évidence le type de relation qui existe entre les deux méthodes de comptage (observation visuelle et automatique) après normalisation des données (logarithmes décimaux) au moyen du logiciel Statview' 1 .

L'analyse de la variance (ANOVA²) a été utilisée afin de comparer les résultats des différents comptages.

\section{3 - RÉSULTATS ET DISCUSSION}

\subsection{Souches bactériennes de référence}

Chaque échantillon est dénombré en microscopie en épifluorescence au moyen de l'analyseur d'images et par observation visuelle. Les résultats des dénombrements et leurs intervalles de confiance obtenus par les deux méthodes de comptage sont présentés dans le Tableau 2. La gamme des abondances bactériennes des échantillons varie de $10^{5}$ à $10^{8}$ cellules $/ \mathrm{ml}$. Cette gamme de concentration tient compte des abondances extrêmes des cellules bactériennes présentes dans les environnements aquatiques étudiés (minima: $1,2110^{5}$ pour l'étang de Thau $n^{\circ} 102$, maxima : $1,8010^{8}$ pour le rejet de la station de lagunage $n^{\circ} 150$ ). La distribution des cellules bactériennes dans les 30 champs comptés à partir desquels la moyenne est calculée présente une répartition soit aléatoire soit régulière qui se traduit par de faibles écarts à la moyenne.

Afin de tester la concordance entre les deux méthodes de dénombrement un modèle de régression linéaire simple est calculé (fig. 2). Le coefficient de corrélation $r$ de ce modèle est de 0,997 avec une probabilité $p=0,0001$, la pente n'est pas significativement différente de 1 et l'ordonnée à l'origine n'est pas significativement différente de 0 .

1. Statview SE 1.03 Abacus Concepts, Inc 1984 Bonita Ave Berkeley CA 94704. 
Tableau 2 Test de répartition $(I(n-1)$, nombre de champs microscopiques dénombrés $n=30$ ), moyennes (cellules $/ \mathrm{ml}$ ) et intervalles de confiance au seuil de signification de $95 \%$ des dénombrements cellulaires de 30 suspensions de souches bactériennes de référence obtenus par analyse d'images (Automatique) et par observation visuelle (Visuel).

Table 2 Dispersion test (I(n-1), number of microscopics fields counted $n=30)$, mean (cells $/ \mathrm{ml})$ and confidence limits $(\rho=0.05)$ of bacterial suspension counts of culture collection samples by image analysis (Automatique) and visual observation (Visuel).

\begin{tabular}{|c|c|c|c|}
\hline $\begin{array}{l}\text { No de souches } \\
\text { de télérence }\end{array}$ & $I(n-1)$ & $\begin{array}{c}\text { Dénonimbrement } \\
\text { Aulomatique (cellules/mi) }\end{array}$ & $\begin{array}{c}\text { Dénombrement } \\
\text { Visual (cellules/ml) }\end{array}$ \\
\hline 1 & 14,6 & $2,19 \pm 0,1110^{7}$ & $2,57 \pm 0,110^{7}$ \\
\hline 2 & 27,9 & $1,01 \pm 0,0710^{6}$ & $1,42 \pm 0,1210^{6}$ \\
\hline 3 & 37,5 & $3,16 \pm 0,2310^{6}$ & $3,24 \pm 0,2510^{6}$ \\
\hline 4 & 16,1 & $5,67 \pm 0,2610^{7}$ & $5,06 \pm 0,210^{7}$ \\
\hline 5 & 24,7 & $2,23 \pm 0,1310^{6}$ & $2,09 \pm 0,1310^{6}$ \\
\hline 6 & 31,9 & $2,68 \pm 0,0810^{5}$ & $2,52 \pm 0,210^{5}$ \\
\hline 7 & 10,5 & $2,78 \pm 0,0910^{6}$ & $2,87 \pm 0,1210^{6}$ \\
\hline 8 & 26,9 & $3,75 \pm 0,0910^{8}$ & $3,50 \pm 0,210^{8}$ \\
\hline 9 & 29,2 & $1,74 \pm 0,0110^{6}$ & $1,92 \pm 0,1710^{6}$ \\
\hline 10 & 37,9 & $8,01 \pm 0,2310^{6}$ & $8,98 \pm 0,7510^{6}$ \\
\hline 11 & 26,7 & $5,90 \pm 0,1810^{7}$ & $6,30 \pm 0,4110^{7}$ \\
\hline 12 & 16,7 & $6,51 \pm 0,5110^{7}$ & $6,62 \pm 0,3310^{7}$ \\
\hline 13 & 26,6 & $7,07 \pm 0,5210^{7}$ & $6,70 \pm 0,4410^{7}$ \\
\hline 14 & 31,2 & $6,76 \pm 0,6210^{7}$ & $6,89 \pm 0,4810^{7}$ \\
\hline 15 & 29,9 & $6,53 \pm 0,6310^{7}$ & $6,51 \pm 0,510^{7}$ \\
\hline 16 & 36,7 & $6,59 \pm 0,3910^{7}$ & $6,70 \pm 0,5510^{7}$ \\
\hline 17 & 34 & $2,97 \pm 0,5510^{8}$ & $1,65 \pm 0,1510^{8}$ \\
\hline 18 & 32,25 & $9,36 \pm 0,7710^{6}$ & $1,06 \pm 0,0610^{7}$ \\
\hline 19 & 23,4 & $3,39 \pm 0,1910^{7}$ & $3,10 \pm 0,210^{7}$ \\
\hline 20 & 28,3 & $4,15 \pm 0,4310^{7}$ & $3,67 \pm 0,2810^{7}$ \\
\hline 21 & 40,9 & $3,35 \pm 0,2610^{8}$ & $3,80 \pm 0,3610^{8}$ \\
\hline 22 & 32,2 & $3,63 \pm 0,2510^{8}$ & $4,14 \pm 0,4710^{8}$ \\
\hline 23 & 34 & $9,67 \pm 0,4210^{8}$ & $9,23 \pm 0,4910^{8}$ \\
\hline 24 & 16,8 & $4,92 \pm 0,2910^{8}$ & $4,58 \pm 0,2610^{8}$ \\
\hline 25 & 33 & $6,27 \pm 0,3410^{7}$ & $6,10 \pm 0,4510^{7}$ \\
\hline 26 & 30 & $3,56 \pm 0,1810^{8}$ & $4,14 \pm 0,2710^{8}$ \\
\hline 27 & 28 & $5,28 \pm 0,3210^{8}$ & $6,00 \pm 0,2210^{8}$ \\
\hline 28 & 17,5 & $7,01 \pm 0,510^{8}$ & $7,91 \pm 0,5410^{8}$ \\
\hline 29 & 19,2 & $7,41 \pm 0,4710^{6}$ & $8,04 \pm 0,6210^{6}$ \\
\hline 30 & 25,7 & $1,94 \pm 0,210^{6}$ & $2,08 \pm 0,1510^{6}$ \\
\hline
\end{tabular}




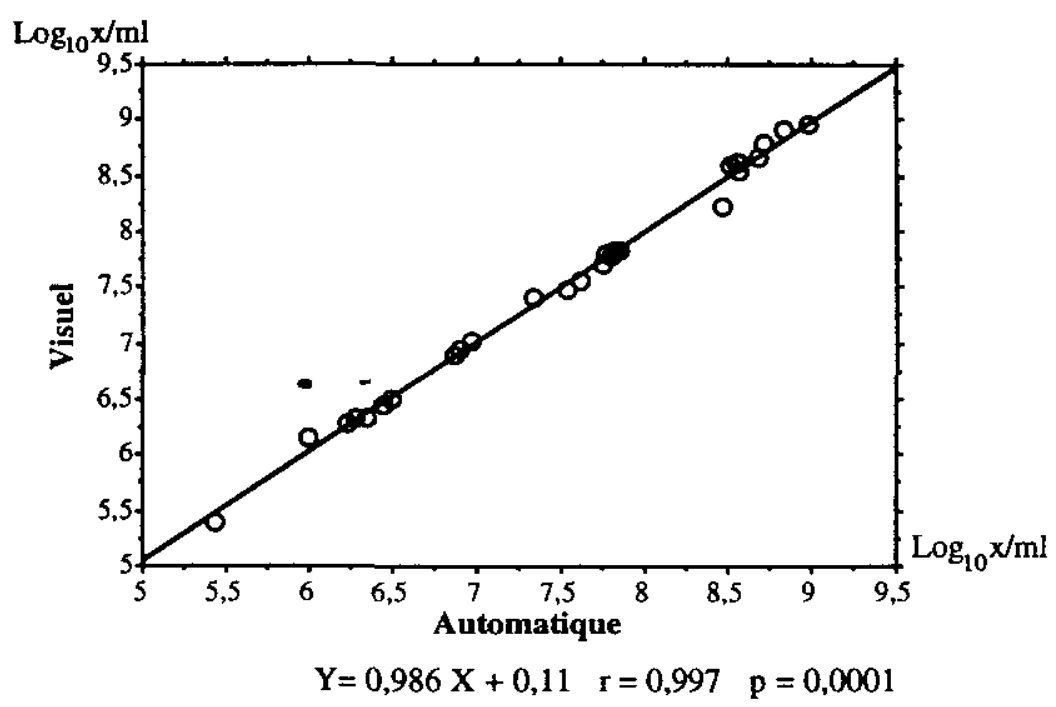

Figure 2 Représentation graphique de la régression linéaire simple calculée à partir des dénombrements bactériens réalisés sur les suspensions de souches de référence par les deux techniques de comptage : Visuel et Automatique.

Linear regression of visual and image analysis automated bacterial counts of culture collection strains : Visuel and Automatique.

\section{2 Échantillons d'eaux}

Les résultats des dénombrement obtenus par les deux méthodes sont présentés dans le Tableau 3. Les distributions des échantillons sont de type aléatoire ou régulier. Tout comme pour les souches bactériennes de référence, une excellente concordance existe entre les deux méthodes de dénombrement.

Cette très bonne relation est visualisée par le calcul du modèle de régression linéaire simple (Fig. 3) dont le coefficient de corrélation $r$ est de 0,996 avec une probabilité $p=0,0001$. La pente de ce modèle n'est pas significativement différente de 1 et son ordonnée à l'origine n'est pas significativement différente de 0 . 
Tableau 3 Test de répartition ( $(n-1)$, nombre de champs microscopiques dénombrés: $n=30$, pour $n=10$ ), moyennes (cellules $/ \mathrm{ml}$ ) et intervalles de confiance au seuil de signification de $95 \%$ des dénombrements cellulaires des échantillons des différents types d'eaux (voir texte 2.1) réalisés par analyse d'images (automatique) et par observation visuelle (Visuel).

Table 3 Dispersion test $(I(n-1)$, number of microscopics fields counted $n=30$, * for $n=10)$, mean $(\mathrm{cel} / \mathrm{s} / \mathrm{ml})$ and confidence limits $(p=0.05)$ of bacterial counts of water samples by image analysis (Automatique) and visual observation (Visuel).

\begin{tabular}{|c|c|c|c|}
\hline $\begin{array}{l}N^{\circ} \text { de souches } \\
\text { de référence }\end{array}$ & $1(n-1)$ & $\begin{array}{l}\text { Dénombrement } \\
\text { Automatique (cellules/ml) }\end{array}$ & $\begin{array}{c}\text { Dénombrement } \\
\text { Visuel (ceilules/mi) }\end{array}$ \\
\hline $101^{*}$ & 4,2 & $7,11 \pm 1,0910^{5}$ & $6,71 \pm 0,610^{5}$ \\
\hline $102^{*}$ & 6,7 & $1,33 \pm 0,1410^{5}$ & $1,21 \pm 0,110^{5}$ \\
\hline $103^{*}$ & 8 & $1,46 \pm 0,1410^{6}$ & $1,53 \pm 0,1210^{6}$ \\
\hline 104 & 44,4 & $1,78 \pm 0,0210^{6}$ & $1,69 \pm 0,0710^{6}$ \\
\hline $105^{*}$ & 2,4 & $1,74 \pm 0,0910^{6}$ & $1,51 \pm 0,110^{6}$ \\
\hline 106 & 32 & $3,05 \pm 0,2810^{6}$ & $3,52 \pm 0,2610^{6}$ \\
\hline 107 & 13 & $4,39 \pm 0,3110^{6}$ & $4,69 \pm 0,2210^{6}$ \\
\hline 108 & 9,9 & $2,5 \pm 0,2810^{6}$ & $2,28 \pm 0,2110^{6}$ \\
\hline $109^{*}$ & 3,09 & $3,55 \pm 0,2610^{6}$ & $3,26 \pm 0,2410^{6}$ \\
\hline $110^{*}$ & 4,53 & $3,95 \pm 0,2310^{6}$ & $3,62 \pm 0,1610^{6}$ \\
\hline $111^{*}$ & 7,01 & $2,08 \pm 0,3110^{6}$ & $2,10 \pm 0,210^{6}$ \\
\hline $112^{*}$ & 3,41 & $4,49 \pm 0,110^{6}$ & $4,59 \pm 0,3210^{6}$ \\
\hline 113 & 11,5 & $1,45 \pm 0,1710^{6}$ & $1,60 \pm 0,1410^{6}$ \\
\hline 114 & 11,7 & $2,54 \pm 0,2310^{6}$ & $2,78 \pm 0,110^{6}$ \\
\hline 115 & 16,65 & $4,09 \pm 0,2910^{6}$ & $4,19 \pm 0,2210^{6}$ \\
\hline 116 & 15,2 & $1,8 \pm 0,0510^{6}$ & $1,72 \pm 0,0710^{6}$ \\
\hline $117^{*}$ & 6,42 & $1,2 \pm 0,1710^{6}$ & $1,29 \pm 0,0710^{6}$ \\
\hline 118 & 13,9 & $2,3 \pm 0,2110^{6}$ & $2,06 \pm 0,3910^{6}$ \\
\hline 119 & 21 & $3,79 \pm 0,3410^{6}$ & $3,38 \pm 0,2510^{6}$ \\
\hline 120 & 15,5 & $1,25 \pm 0,1910^{6}$ & $1,42 \pm 0,110^{6}$ \\
\hline 121 & 17,6 & $1,29 \pm 0,210^{6}$ & $1,43 \pm 0,1510^{6}$ \\
\hline $122^{*}$ & 4,38 & $1,54 \pm 0,1310^{6}$ & $1,32 \pm 0,1110^{6}$ \\
\hline $123^{*}$ & 4,59 & $2,34 \pm 0,210^{6}$ & $2,53 \pm 0,1310^{6}$ \\
\hline 124 & 5,7 & $1,4 \pm 0,1110^{6}$ & $1,44 \pm 0,2310^{6}$ \\
\hline $125^{*}$ & 4,99 & $2,53 \pm 0,2210^{6}$ & $2,72 \pm 0,1910^{6}$ \\
\hline 126 * & 4,71 & $1,16 \pm 0,1310^{6}$ & $1,01 \pm 0,1810^{6}$ \\
\hline 127 & 18,6 & $2,47 \pm 0,1510^{6}$ & $2,49 \pm 0,1510^{6}$ \\
\hline 128 & 19 & $1,88 \pm 0,0610^{6}$ & $1,99 \pm 0,1110^{6}$ \\
\hline 129 & 13,7 & $1,79 \pm 0,0310^{6}$ & $1,75 \pm 0,0410^{6}$ \\
\hline 130 & 27,5 & $1,79 \pm 0,0210^{6}$ & $1,82 \pm 0,0410^{6}$ \\
\hline 131 & 18,15 & $3,32 \pm 0,3210^{5}$ & $3,57 \pm 0,2610^{5}$ \\
\hline 132 & 18,4 & $3,47 \pm 0,2910^{5}$ & $3,42 \pm 0,2510^{5}$ \\
\hline $133^{*}$ & 6,3 & $1,98 \pm 0,1210^{5}$ & $1,76 \pm 0,110^{5}$ \\
\hline 134 & 23,1 & $2,81 \pm 0,3310^{5}$ & $2,39 \pm 0,1110^{5}$ \\
\hline 135 & 17,3 & $3,94 \pm 0,2610^{5}$ & $3,60 \pm 0,5910^{5}$ \\
\hline
\end{tabular}




\begin{tabular}{|cccc|}
\hline $\begin{array}{c}N^{\circ} \text { de sauches } \\
\text { de référence }\end{array}$ & $\mathbf{l}(\mathbf{n}-\mathbf{1})$ & $\begin{array}{c}\text { Dénombrement } \\
\text { Automatique (cellules/m) }\end{array}$ & $\begin{array}{c}\text { Dénombrement } \\
\text { Vlsuel (cellufes/ml) }\end{array}$ \\
\hline 136 & 17,2 & $2,14 \pm 0,1210^{5}$ & $2,13 \pm 0,1110^{5}$ \\
137 & 20,8 & $1,52 \pm 0,0810^{6}$ & $1,51 \pm 0,0910^{6}$ \\
138 & 43,9 & $1,42 \pm 0,1410^{5}$ & $1,66 \pm 0,1110^{5}$ \\
139 & 21,9 & $3,08 \pm 0,0810^{7}$ & $2,91 \pm 0,1210^{7}$ \\
140 & 30,5 & $1,99 \pm 0,1410^{5}$ & $1,83 \pm 0,1110^{5}$ \\
141 & 18,3 & $1,29 \pm 0,0710^{6}$ & $1,15 \pm 0,1410^{6}$ \\
142 & 15,8 & $1,53 \pm 0,0710^{6}$ & $1,21 \pm 0,1310^{6}$ \\
143 & 26,1 & $-3,14 \pm 0,1810^{7}$ & $3,05 \pm 0,2910^{7}$ \\
144 & 34,6 & $2,54 \pm 0,1710^{7}$ & $2,09 \pm 0,1810^{7}$ \\
145 & 21,6 & $2,05 \pm 0,1210^{7}$ & $1,74 \pm 0,210^{7}$ \\
146 & 17,2 & $4,79 \pm 0,2910^{5}$ & $4,30 \pm 0,6610^{5}$ \\
147 & 36,9 & $9,14 \pm 0,5910^{5}$ & $7,80 \pm 0,9510^{5}$ \\
148 & 37,1 & $1,31 \pm 0,110^{6}$ & $1,25 \pm 0,1710^{6}$ \\
149 & 42,4 & $1,74 \pm 0,1210^{6}$ & $1,44 \pm 0,0710^{6}$ \\
150 & 7,1 & $1,56 \pm 0,1510^{8}$ & $1,80 \pm 0,0910^{8}$ \\
\hline
\end{tabular}

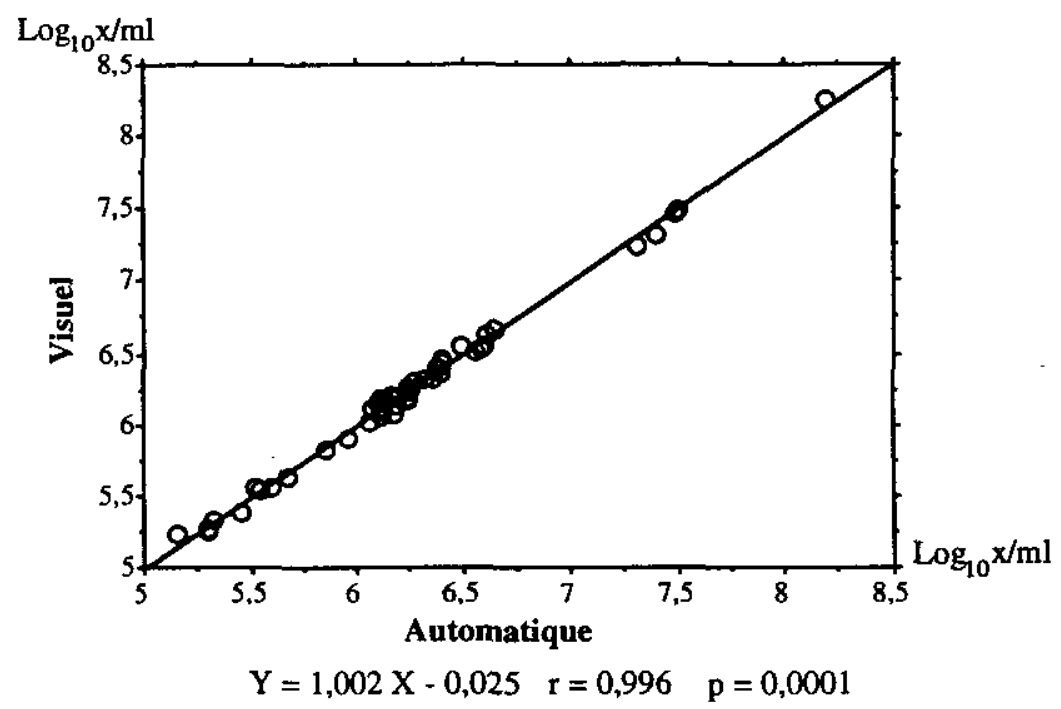

Figure 3 Représentation graphique de la régression linéaire simple calculée à partir des dénombrements bactériens réalisés sur les échantillons d'eaux par les deux techniques de comptage: Visuel et Automatique.

Linear regression of visual and image analysis automated bacterial counts of water samples: Visuel and Automatique. 


\subsection{Variabilité}

Si la technique d'analyse d'images automatisée permet de compter de façon reproductible et objective les échantillons, le dénombrement par un observateur est obligatoirement subjectif. Pour évaluer cette subjectivité, des dénombrements ont été menés en parallèle en observation visuelle par deux opérateurs expérimentés puis la variabilité du dénombrement entre les deux opérateurs et le comptage automatique a été comparée. Dix huit échantillons pris au hasard (6 échantillons de souches de référence et 12 échantillons de différents types d'eau) ont éfé dénombrés de façon automatique et de façon visuelle par deux opérateurs (opérateur $n^{\circ} 1$ et opérateur $n^{\circ} 2$ ). Les résultats sont présentés dans le Tableau 4. L'analyse de variance ne donne pas de différence significative entre les trois façons de dénombrer au seuil de probabilité de $99 \%$. Les moyennes calculées à partir des 18 échantillons sont très proches: $6,09,6,08$ et 6,11 respectivement pour la méthode automatique, l'opérateurs $n^{\circ} 1$ et l'opérateur $n^{\circ} 2$. La valeur obtenue avec le comptage automatisé se situe entre les valeurs obtenues par les deux opérateurs en observation visuelle. Les différences moyennes calculées entre les trois méthodes mettent en évidence que; bien que non significative, la plus élevée $(0,03)$ est enregistrée entre les comptages effectués par l'opérateur $n^{\circ} 1$ et l'opérateur $n^{\circ} 2$ alors que cette différence n'est que de 0,01 et 0,02 quand le dénombrement par la méthode automatique est comparé avec ceux obtenus avec les opérateurs $n^{\circ} 1$ et $n^{\circ} 2$.

\section{4 - CONCLUSION}

Le comptage par microscopie en épifluorescence couplé à un système d'analyse d'images permet aux utilisateurs de s'affranchir des aspects les plus fastidieux et coûteux en temps de cette technique indispensable dans l'estimation des abondances bactériennes totales des échantillons d'eaux. II assure une reproductibilité du comptage comparable à celui réalisé par deux manipulateurs expérimentés et de ce fait contribue à la standardisation des procédures de comptage. La rapidité de la saisie des champs-images accroît les capacités d'analyse et donc diminue l'effort analytique des nombreux échantillons qu'il est indispensable de traiter dans une étude d'écologie bactérienne. Enfin le stockage informatique des champs-images dénombrés permet a posteriori d'accéder à des informations d'ordre qualitatif complémentaires du dénombrement total. Tel est le cas du double marquage des cellules dont la saisie instantanée des images et la sensibilité de la caméra permettent de passer outre le phénomène d'extinction propre à certains fluorochromes. L'utilisation d'un analyseur d'images performant tel que celui testé au cours de cette étude constitue un complément analytique essentiellement objectif pour toutes les études du compartiment bactérien qui reposent sur les techniques de microscopie en épifluorescence. 
Tableau 4 Test de répartition $(I(n-1)$, nombre de champs microscopiques dénombrés $n=30$ ), moyennes (cellules $/ \mathrm{ml}$ ) et intervalles de confiance au seuil de signification de $95 \%$ des dénombrements bactériens de 18 suspensions ou échantillons (pris au hasard parmis les 80 ) obtenus par analyse d'images (automatique) et par observation visuelle de l'opérateur $n^{\circ} 1$ et de l'opérateur $n^{\circ} 2$. (2...) voir Tableau 2, (104...) voir Tableau 3.

Table 4 Dispersion test $(I(n-1)$, number of microscopics fields counted $n=30)$, mean $(c e l l s / m l)$ and confidence limits $(p=0,05)$ of bacterial counts of 18 random samples by image analysis and visual observation of two experimenters (" opérateurs $n^{\circ} 1 "$, "opérateur $\left.n^{\circ} 2 "\right)$. (2...) see Table 2, (104...) see Table 3.

\begin{tabular}{|c|c|c|c|c|}
\hline \multirow[b]{2}{*}{ Echantillons } & \multirow[b]{2}{*}{$I(n-1)$} & \multicolumn{3}{|c|}{ Dénombrements (cellules/ml) } \\
\hline & & $\begin{array}{l}\text { Automatique } \\
\text { Mudicam @ }\end{array}$ & $\begin{array}{c}\text { Visuel } \\
\text { Opérateur nº } 1\end{array}$ & $\begin{array}{c}\text { Visuel } \\
\text { Opérateur } n^{\circ} 2\end{array}$ \\
\hline Proteus vulgaris (2) & 27,9 & $1,01 \pm 0,0710^{6}$ & $1,42 \pm 0,1110^{6}$ & $1,60 \pm 0,1710^{6}$ \\
\hline Salmonella anizonae (3) & 37,5 & $3,16 \pm 0,2310^{6}$ & $3,24 \pm 0,2510^{6}$ & $3,00 \pm 0,210^{6}$ \\
\hline Salmonella typhimurium (4) & 16,1 & $5,67 \pm 0,2610^{7}$ & $5,06 \pm 0,210^{7}$ & $5,07 \pm 0,2310^{7}$ \\
\hline Proteus vulgaris (5) & 24,7 & $2,23 \pm 0,1310^{6}$ & $2,09 \pm 0,1310^{6}$ & $2,30 \pm 0,0610^{6}$ \\
\hline Eau saumấtre (104) & 44,4 & $1,78 \pm 0,0210^{6}$ & $1,69 \pm 0,0710^{6}$ & $1,37 \pm 0,1310^{6}$ \\
\hline Eau de mer $(1 \pm 6)$ & 15,2 & $1,80 \pm 0,0510^{6}$ & $1,72 \pm 0,0710^{6}$ & $2,00 \pm 0,1610^{6}$ \\
\hline Fletve Rhơre (127) & 18,6 & $2,47 \pm 0,1510^{6}$ & $2,49 \pm 0,1510^{6}$ & $2,72 \pm 0,2610^{6}$ \\
\hline Escherichia coli 1832 (6) & 31,9 & $2,68 \pm 0,0810^{5}$ & $2,52 \pm 0,210^{5}$ & $3,00 \pm 0,210^{5}$ \\
\hline Heuve Rhône (129) & 13,7 & $1,79 \pm 0,0310^{6}$ & $1,75 \pm 0,0410^{6}$ & $1,92 \pm 0,1310^{6}$ \\
\hline Serratia marcescens ( 7 ) & 10,5 & $2,78 \pm 0,0910^{6}$ & $2,87 \pm 0,1210^{6}$ & $2,70 \pm 0,1710^{6}$ \\
\hline Fleuve Hérault (131) & 18.1 & $3,32 \pm 0,3210^{5}$ & $3,57 \pm 0,2610^{5}$ & $3,50 \pm 0,3410^{5}$ \\
\hline Fleuve Hérault (132) & 18,4 & $3,47 \pm 0,2910^{5}$ & $3,42 \pm 0,2510^{5}$ & $3,50 \pm 0,3410^{5}$ \\
\hline Eau d'égout (134) & 23,1 & $2,81 \pm 0,3310^{5}$ & $2,39 \pm 0,1110^{5}$ & $2,65 \pm 0,3410^{5}$ \\
\hline $\begin{array}{l}\text { Sortie de station d'épuration } \\
\text { (136) }\end{array}$ & 17,2 & $2,14 \pm 0,1210^{5}$ & $2,13 \pm 0,1110^{5}$ & $2,23 \pm 0,1710^{5}$ \\
\hline $\begin{array}{l}\text { Sortie de station d'épuration } \\
\text { (137) }\end{array}$ & 20,8 & $1,52 \pm 0,0810^{6}$ & $1,51 \pm 0,0910^{6}$ & $1,77 \pm 0,1310^{6}$ \\
\hline $\begin{array}{l}\text { Sortie de station d'épuration } \\
\text { (138) }\end{array}$ & 43,9 & $1,42 \pm 0,1410^{5}$ & $1,66 \pm 0,1110^{5}$ & $1,93 \pm 0,0110^{5}$ \\
\hline $\begin{array}{l}\text { Sortie de station d'épuration } \\
\text { (139) }\end{array}$ & 21,9 & $3,08 \pm 0,0810^{7}$ & $2,91 \pm 0,1210^{7}$ & $3,20 \pm 0,2410^{7}$ \\
\hline $\begin{array}{l}\text { Sortie de station d'épuration } \\
\text { (140) }\end{array}$ & 30,5 & $1,99 \pm 0,1410^{5}$ & $1,83 \pm 0,1110^{5}$ & $2,38 \pm 0,0210^{5}$ \\
\hline
\end{tabular}




\section{RÉFÉRENCES BIBLIOGRAPHIQUES}

ABGRALL B., BOURGEOIS C.M., 1989. Dénombrement de la flore totale de produits alimentaires par la technique DEFT. Sci. Aliments, 9, 713-724.

BJORNSEN P.K., 1986. Automatic determination of bacterioplankton biomass by image analysis. Appl. Environ. Microbid., 51, 1199-1204.

CHERYL M., DAVIES WRc., 1991. A comparison of fluorochromes for direct viable counts by image analysis. Letters in Appl. Microbiol., 13, 58-61.

DASEN A., PITON C., BEUVIER E., GUERRY P., 1989. Numération des cellules somatiques du lait cru par la technique DEFT associée à un comptage visuel ou par anałyse d'image. Lait, 69, 461-477.

ELLIOTT J.M., 1977. Some methods for the statistical analysis of samples of benthic invertebrates. $2^{\text {nd }}$ edition Freshwater biological association scientific publication $n^{\circ} 25$, Ferry House, $157 \mathrm{pp}$.

FRY J.C., 1988. Determination of biomass. In: Methods in aquatic bacteriology. Austin B. (ed). John Wiley et Sons, Chichester, 27-72.

GRAPPIN R., JEUNET R., 1974. Premiers essais de l'appareil Fossomatic pour la détermination automatique du nombre de cellules du lait. Lait, 54, 627-644.

HARA S., TERAUCHI K. and KOIKE I., 1991. Abundance of viruses in marine waters: Assessment by epifluorescence and transmission electron microscopy. Appl. Environ. Microbiol., 57, 2731-2734.

JAEGGI N.E., SIMES V. and HUGUES D., 1989. Evaluation of a television image analyser as an aid to estimation of microbial numbers in food using the direct epifluorescent filter technique. Food Microbiol., 6 , 85-91.

KING L.K., PARKER B.C., 1988. A simple method for enumerating total viable and metabolically active bacteria in groundwater. Appl. Environ. Microbiol., 54, 1630 1631.

OGER C., HERNANDEZ J.F., DELATTRE J.M. DELABROISE A.H. et KRUPSY S., 1987. Etude par épifluorescence de la microflore totale dans une eau minérale embouteillée. Wat. Res. 21 (4), 469-474.

PETTIPHER G.L., RODRIGUES U.M., 1982. Semi-automated counting of bacteria and somatic cells in milk using epifluorescence microscopy and television image analysis. J. Appl. Bacteriol. 53, 323-329.

PORTER K.G., FEIG Y.S., 1980. The use of DAPI for identifying and counting aquatic microflora. Limnol. Oceanogr., 25, 943-948.

ROBARTS R.D., SEPHTON L.M., 1981. The enumeration of aquatic bacteria using DAPI. J. Limnol. Soc. Sth. Afr., 7 (2), 72-74.

ROBERTSON B.R., BUTTON D.K., 1989. Characterizing aquatic bacteria according to population, cell size, and apparent DNA content by flow cytometry. Cytometry, 10, 70-76.

SIERACKI M.E., JOHNSON P.W. and SIEBURTH J.MCN., 1985. Detection, enumeration, and sizing of planktonic bacteria by image-analysed epifluorescence micros copy. Appl. Environ. Microbiol., 49 (4), 799 810.

SIERACKI M.E., REICHENBACH S.E. and WEBB K.L., 1989. Evaluation of automated threshold selection methods for accurately sizing microscopic fluorescent cell by image analysis. Appl. Environ. Microbiol., 55 (11), 2762-2772.

SINGH A., FEJ-PENG Y. and MCFETERS G.A., 1990. Rapid detection of chlorine-induced bacterial injury by the direct viable count method using image analysis. $A p p l$. Environ. Microbiol., 56 (2), 389-394.

STRUGGER R. (1948). Fluorescence microscope examination of bacteria in soil. Can. J. Res. series C., 26, 188-193.

TROUSSELLIER M., ALBAT M., ANDRE P., BALEUX B., 1985. Dénombrements directs des bactéries dans les milieux aquatiques par microscopie en épifluorescence : distribution ef précision des mesures. Rev. Franç. des Sciences de l'Eau, 74, 35-49.

VAN WAMBEKE F., 1988. Numération et taille des bactéries planctoniques au moyen de l'analyse d'images couplée à l'épifluorescence. Ann. Inst. Pasteur/Microbiol., 139, 261-272. 
VECHT-LIFSHITZ S., ISON A.P., 1992. Biotechnological applications of image analysis : present and future prospects. Journal of Biotechnol., 23, 1-18.

YANAGIDA M., MORIKAWA K., HIRAOKA Y., MATSUMOTO S., UEMURA T. and OKADA
S., 1986. Video-connected fluorescence microscopy of large DNA molecules, chromatin and chromosomes. In: Applications of fluorescence in the biomedical sciences. Taylor D.L., Waggoner A.S., Murphy R.F., Lanni F. and Birge R.R. (eds). Alan R. Liss, Inc., New-York, 321-345. 